Supporting Information

\title{
Facile and purification-free synthesis of nitrogenated amphiphilic graphitic carbon dots
}

\author{
Byung Joon Moon, ${ }^{1 \dagger}$ Yelin Oh, ${ }^{1 \dagger}$ Dong Heon Shin, ${ }^{1}$ Sang Jin Kim, ${ }^{1}$ \\ Sanghyun Lee, ${ }^{1,2}$ Tae-Wook Kim, ${ }^{1,2}$ Min Park ${ }^{1}$ and Sukang Bae ${ }^{1 *}$
}

Table of Contents

1. Experimental Section.

2. Visual dispersibility test for N-GCDs.

3. Typical XPS survey spectrum of N-GCDs.

4. High resolution NEXAFS spectrum of N-GCDs.

5. PL mechanism of N-GCDs.

6. Measurement of fluorescence quantum yield of N-GCDs.

7. Stability test of N-GCDs solution using PL measurement system.

8. Schematic illustration of an inverted type polymer-fullerene BHJ solar cell

9. Absorbance of the ITO/PEIE/PTB7:PC ${ }_{71} \mathrm{BM}$ and ITO/PEIE/PTB7:PC ${ }_{71} \mathrm{BM}+2$ wt\%

N-GCDs films

10. Ultraviolet photoelectron spectra (UPS) of the N-GCDs sample.

11. Absorbance of the photo-active materials and PL emission of N-GCDs

12. Time-resolved PL of N-GCDs.

13. Device performance of inverted polymer solar cells with/without N-GCDs.

14. Stability tests of polymer solar cells with/without N-GCDs in PTB7:PC ${ }_{71} B M$ blend system under ambient conditions. 


\section{Experimental Section}

\section{Preparation of N-GCDs}

The N-GCDs were prepared by thermal treatment of fumaronitrile powder (FN) purchased from Tokyo Chemical Industry Co., Ltd. In a typical procedure, 0.3g FN dispersed in $10 \mathrm{~mL}$ DI water was injected into a $50 \mathrm{~mL}$ round bottle flask, and then heated to $225^{\circ} \mathrm{C}$ for 10 min using a hot plate while continually stirring to obtain stable and uniformed products. In consequence, the color of the liquid was changed from colorless to pale yellow, indicating that N-GCDs are successfully formed. After removing excess DI-water using vacuum evaporator, the final product (N-GCDs) was collected after drying process.

\section{Instrumentation}

Atomic force microscopy (AFM) imaging was performed in air using an NX-10 (Park systems). High resolution transmission electron microscopy (HRTEM) image of NGCDs were obtained with a Tecnai G2 F20 operating at 200kV. UV-vis absorption and photo-luminescence (PL) were characterized by Jasco V-670 spectrophotometer and Horiba NanoLog-C, respectively. The Fourier transform infrared (FT-IR) spectrum was obtained on a FT-IR spectrophotometer (Jasco FT/IR-6600). The Raman spectra were obtained using a Horiba Jobin Yvon-Labram HR UV-VisibleNIR Raman microscope spectrometer. X-ray photoelectron spectroscopy was carried out using monochromated Al K $\alpha$ X-ray photons $(h v=1486.6 \mathrm{eV})$. To measure the electron lifetime of N-GCDs, time resolved photoluminescence (TRPL) was performed. The second harmonic $(\mathrm{SHG}=360 \mathrm{~nm})$ of a tunable Ti:sapphire laser 
(MaiTai, Spectra Physics) with an approximate $\sim 100$ fs pulse width and $80 \mathrm{MHz}$ repetition rate was used as the excitation source.

\section{Device Fabrication and Measurement}

Solar cell devices were fabricated on $1 \times 1 \mathrm{~cm}$ pre-patterned ITO coated glass substrates. The ITO-coated glass substrates were cleaned with ethanol, acetone and 2propanol. The ITO-coated glass was treated with $\mathrm{O}_{2}$ plasma for $40 \mathrm{sec}$. PEIE solution was spin-coated with $7 \mathrm{~nm}$ thickness and the PEIE film was baked at $110{ }^{\circ} \mathrm{C}$ for 15 min under ambient conditions. After cooling, the substrates were then transferred into a nitrogen-filled glove box. The blend of PTB7:PC ${ }_{71} \mathrm{BM}(1: 1.5$ (by weight)) with $2 \mathrm{wt} \%$ N-GCDs ternary blend solution was spin-coated onto the PEIE layer to form a $110 \mathrm{~nm}$ thick active layer. Finally, $\mathrm{MoO}_{3}(10 \mathrm{~nm})$ and $\mathrm{Ag}(80 \mathrm{~nm})$ were sequentially deposited on the active layer by thermal evaporation in a vacuum of $6 \times 10^{-7}$ Torr, which defines the active area of $0.0464 \mathrm{~cm}^{2}$. The un-encapsulated solar cells were tested under ambient conditions using a Keithley 2400 SMU and an Oriel xenon lamp (450 W) with an AM 1.5 G filter. A mask was used to define the device illumination area of $0.0464 \mathrm{~cm}^{2}$ to minimize photocurrent generation from the edge of the electrodes. The solar cells (with no protective encapsulation) were then tested in air under an AM 1.5 $\mathrm{G}$ illumination of $100 \mathrm{~mW} \mathrm{~cm} \mathrm{~m}^{-2}$ (Oriel $1 \mathrm{~kW}$ solar simulator), which was calibrated with the International System of Units (SI) (SRC-1000-TC-KG5-N, VLSI Standards, Inc) for accurate measurement. The external quantum efficiency (EQE) was measured using a Oriel IQE-200 (NewPort), a calibrated Si UV detector, and an SR8570 low noise current amplifier. 
2. Visual dispersibility test for N-GCDs

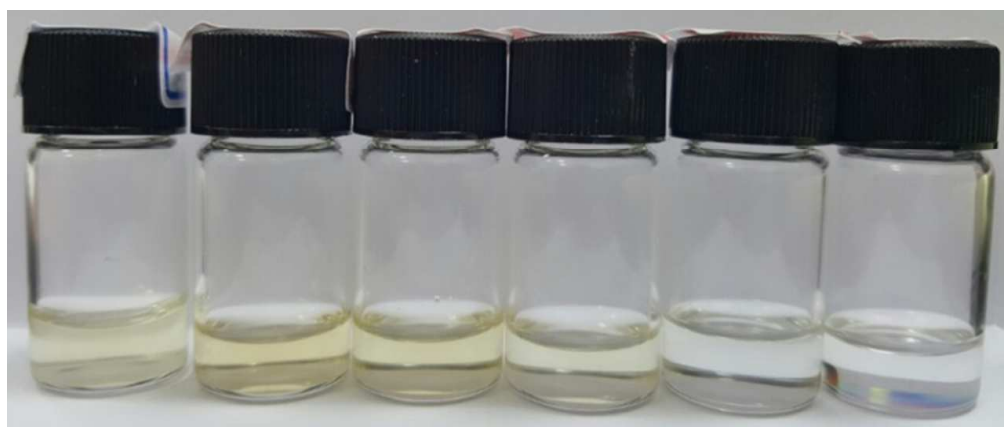

Fig. S1 The visual dispersibility test for N-GCDs (from left: DI-water, ethanol, DMF, THF, chloroform and chlorobenzene). Each sample (conc.: $0.1 \mathrm{mg} / \mathrm{mL}$ ) was sonicated for $5 \mathrm{sec}$ and allowed to settle for $120 \mathrm{hr}$. 
3. Typical XPS survey spectrum of N-GCDs

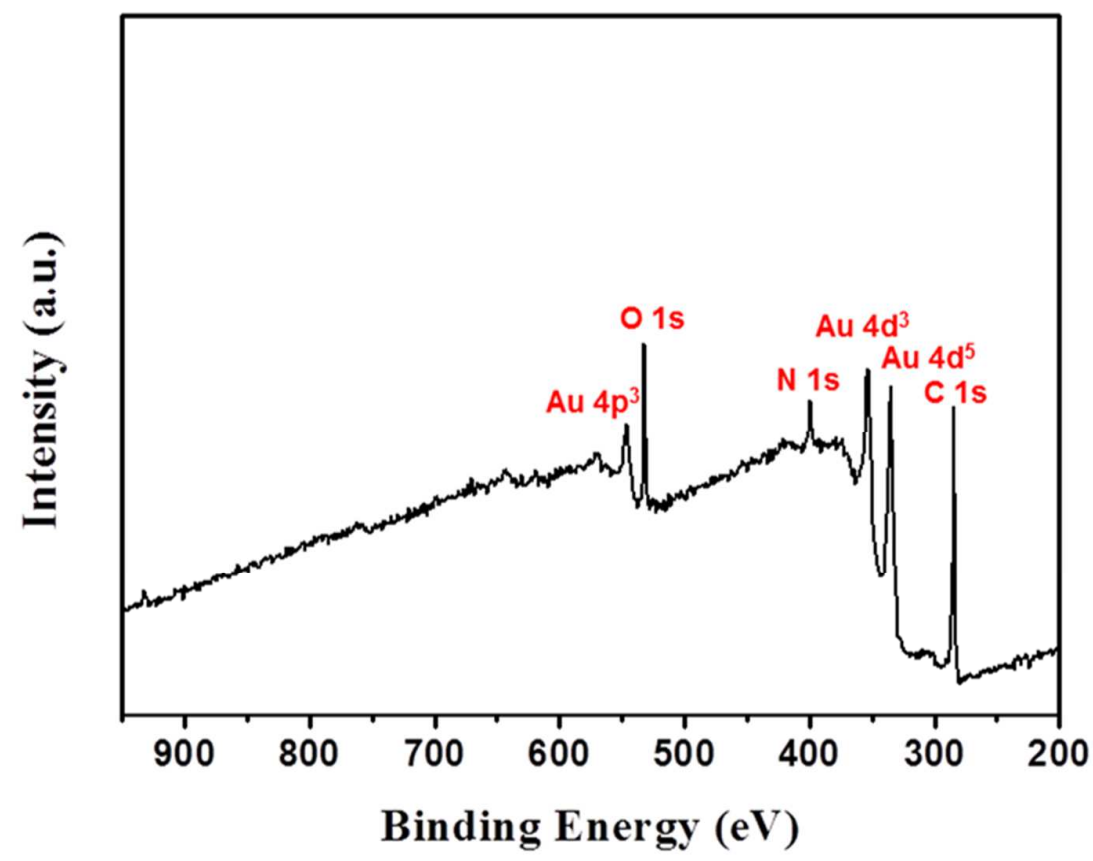

Fig. S2 Typical XPS survey spectrum of N-GCDs on $\mathrm{Si} / \mathrm{SiO}_{2} / \mathrm{Cr} / \mathrm{Au}$ substrate. 


\section{High resolution NEXAFS spectrum of N-GCDs}

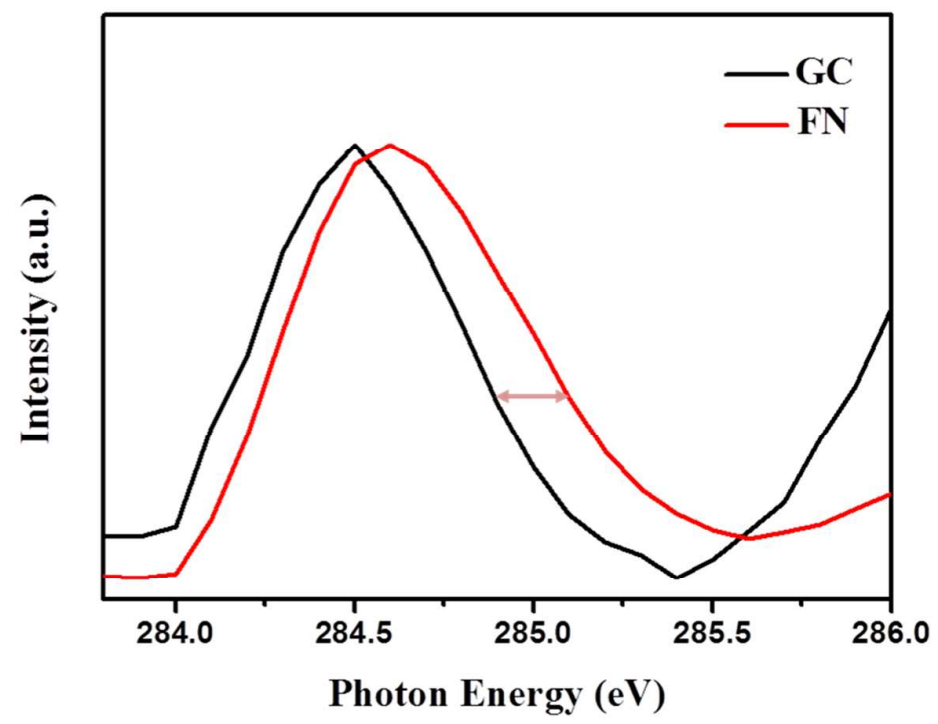

Fig. S3 C K-edge NEXAFS spectra of two different kinds of carbon based QDs. Each spectrum was obtained at $54.7^{\circ}$ incidence of the X-ray beam.

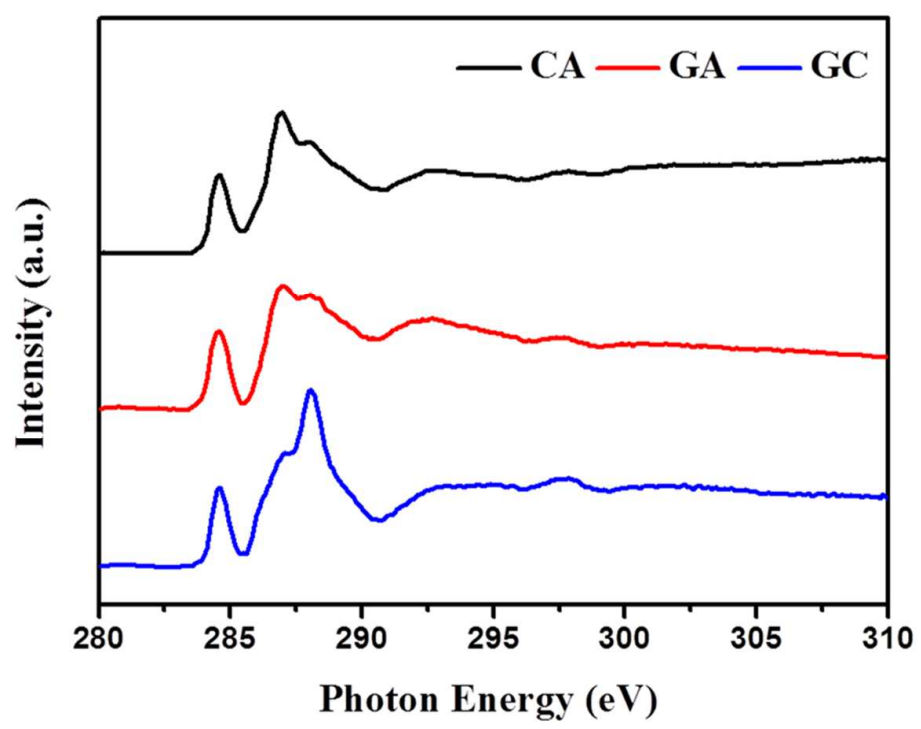

Fig. S4 Overlay plot of the normalized NEXAFS spectra taken at the C K-edge for various carbon based QDs prepared from different organic precursors. Each spectrum was secured at $54.7^{\circ}$ incidence of the X-ray beam. 


\section{PL mechanism of N-GCDs}

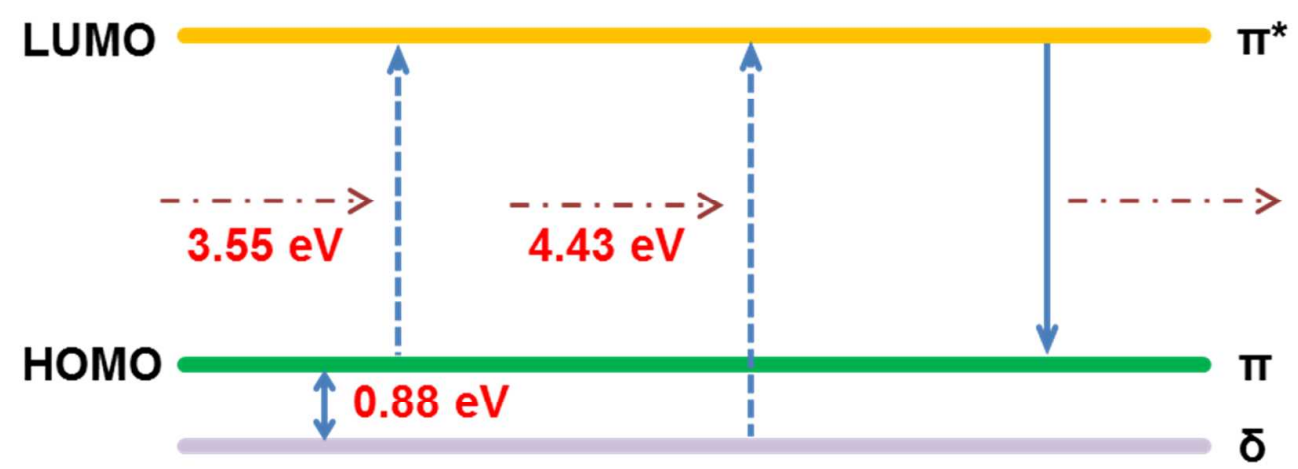

Fig. S5 Typical electronic transitions of triple carbenes at zigzag sites observed in the optical spectra. 


\section{Measurement of fluorescence quantum yield of N-GCDs}

(a)

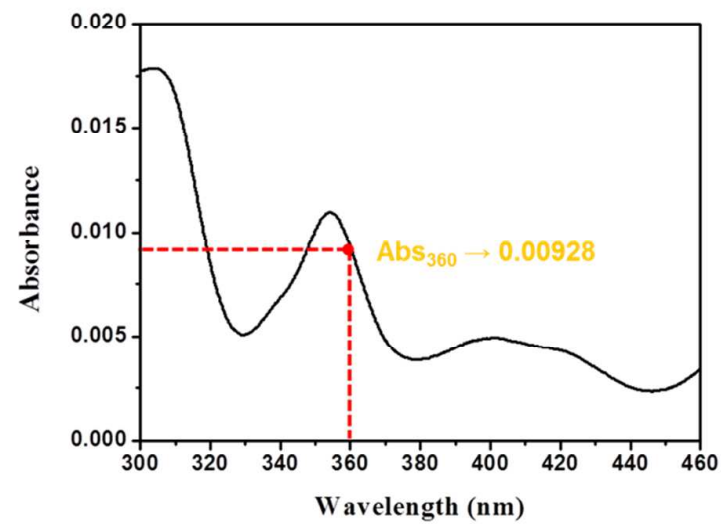

(c)

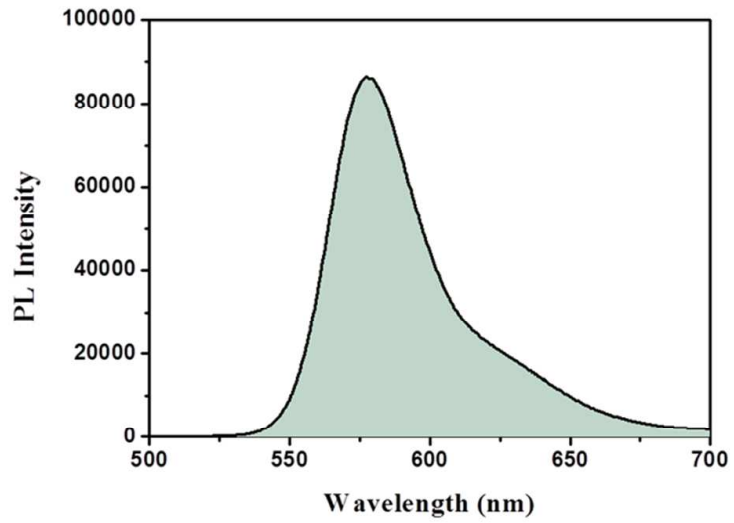

(b)

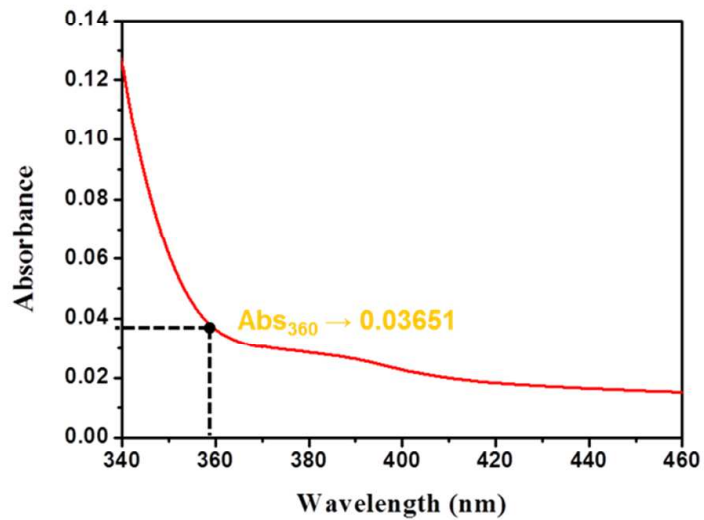

(d)

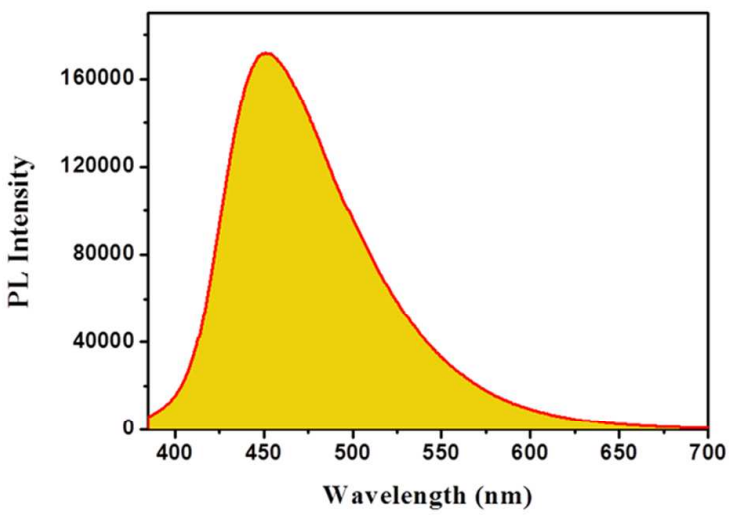

Fig. S6 UV-vis absorption spectra of the Rhodamine B (a) and N-GCDs (b). PL spectra of the Rhodamine B (c) and N-GCDs (d). 
7. Stability test of N-GCDs solution using PL measurement system.

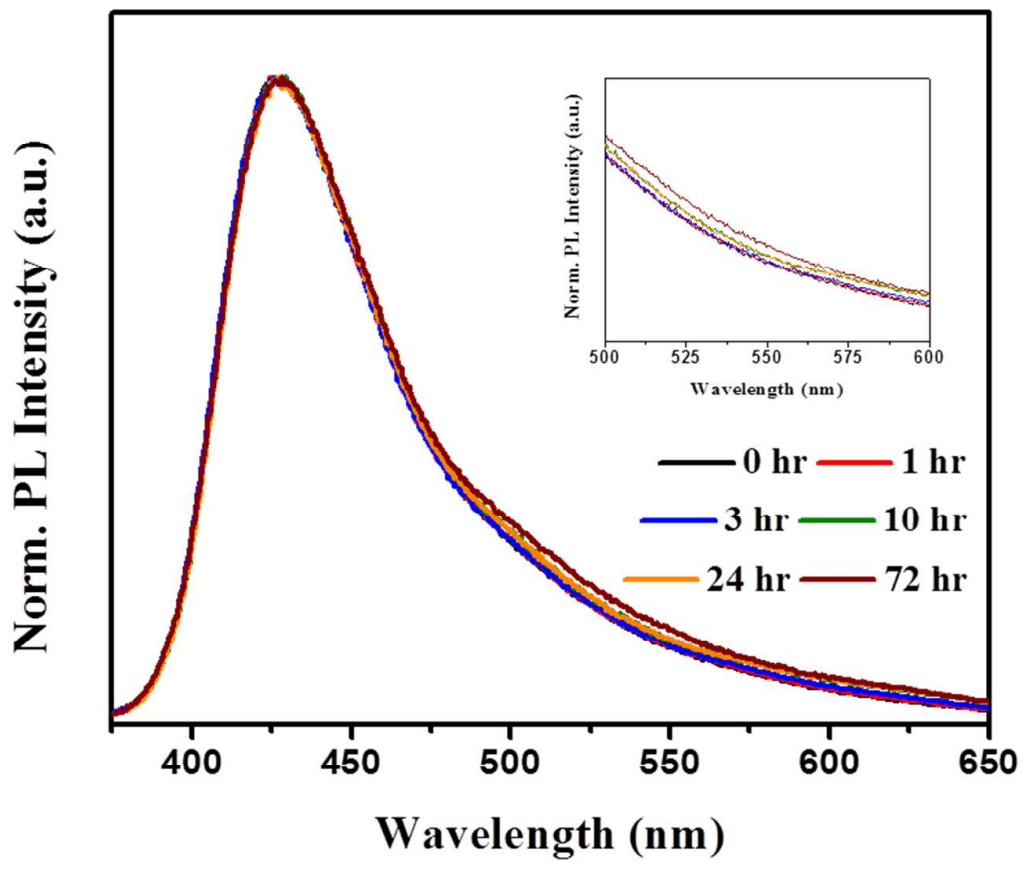

Fig. S7 PL spectra of N-GCDs dissolved in THF. The PL spectra were obtained for excitation at $350 \mathrm{~nm}$ during 3 days. 


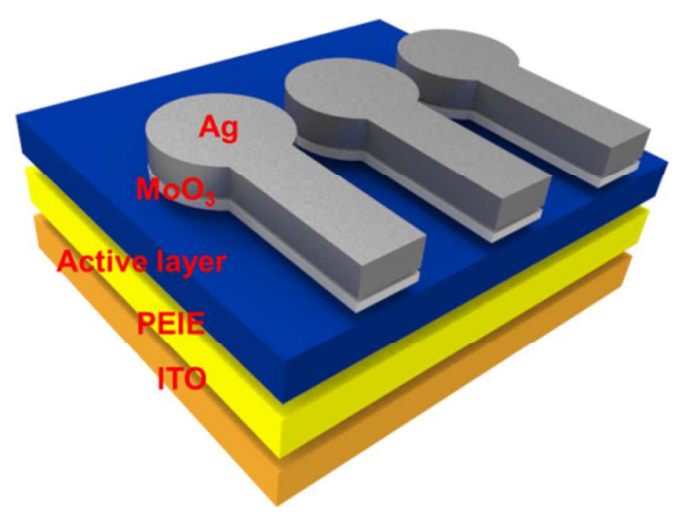

Fig. S8 Schematic illustration of the iPSC structure with the configuration of ITO/PEIE/Active layer (PTB7:PC $71 \mathrm{BM}) / \mathrm{MoO}_{3} / \mathrm{Ag}$.

9. Absorbance of the ITO/PEIE/PTB7:PC 71 BM and ITO/PEIE/PTB7:PC ${ }_{71} B M+$ 2 wt\% N-GCDs films

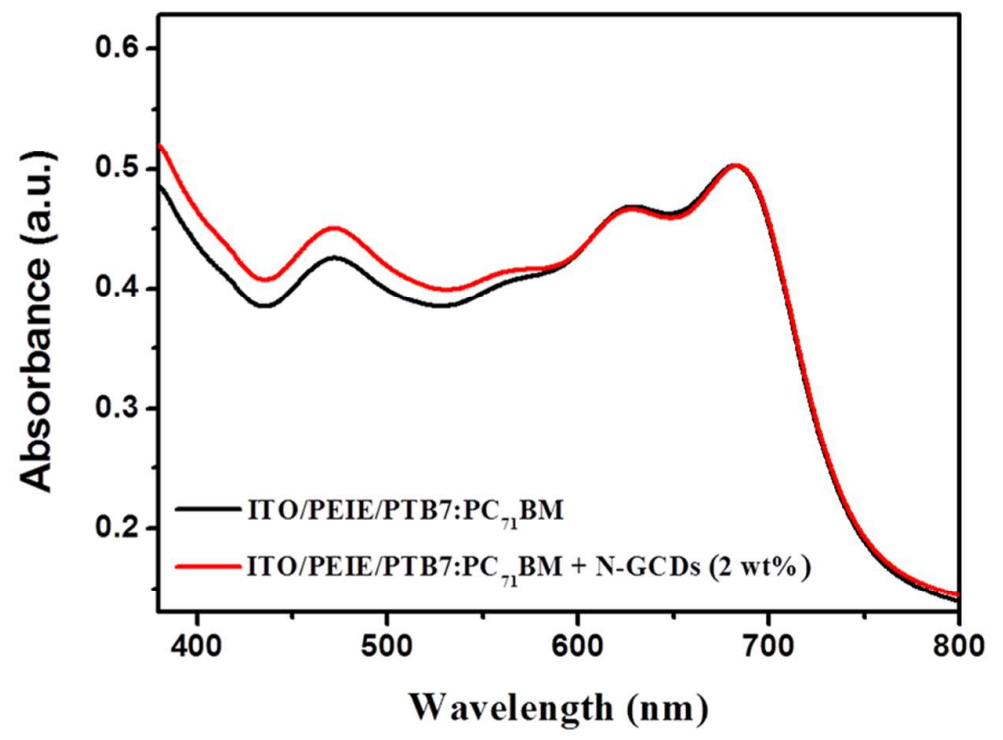

Fig. S9 UV-Vis absorption spectra of the PEIE/PTB7:PC ${ }_{71} \mathrm{BM}$ and PEIE/PTB7:PC ${ }_{71} \mathrm{BM}+\mathrm{N}-\mathrm{GCDs}(2 \mathrm{wt} \%)$ films on ITO substrates. 
10. Ultraviolet photoelectron spectra (UPS) of the N-GCDs sample.

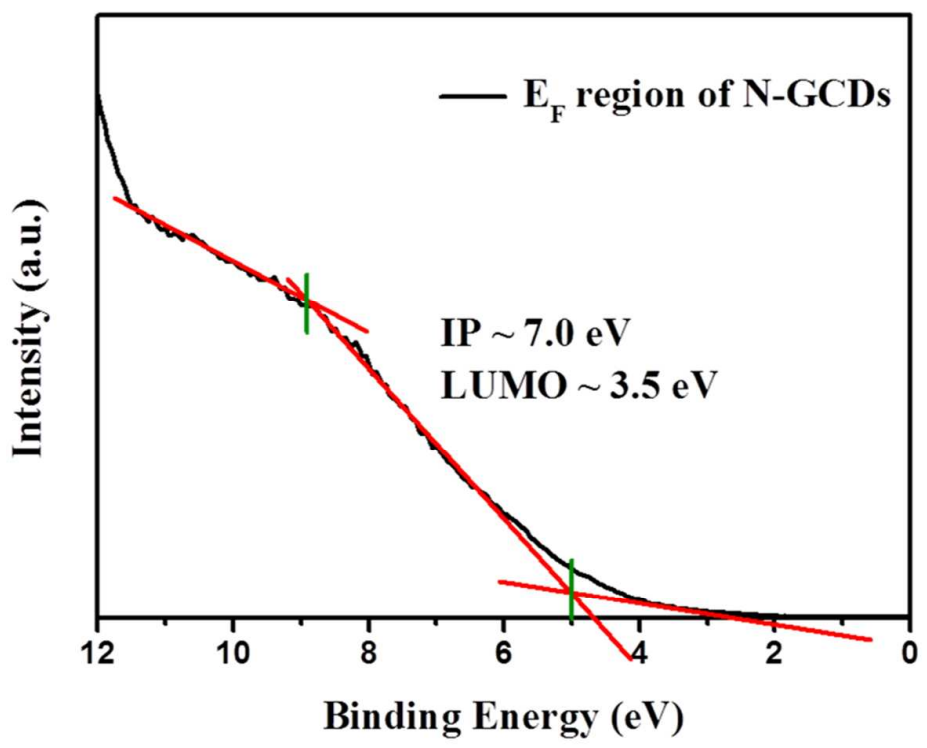

Fig. S10 UPS spectra of the N-GCDs sample.

11. Absorbance of the photo-active materials and PL emission of N-GCDs

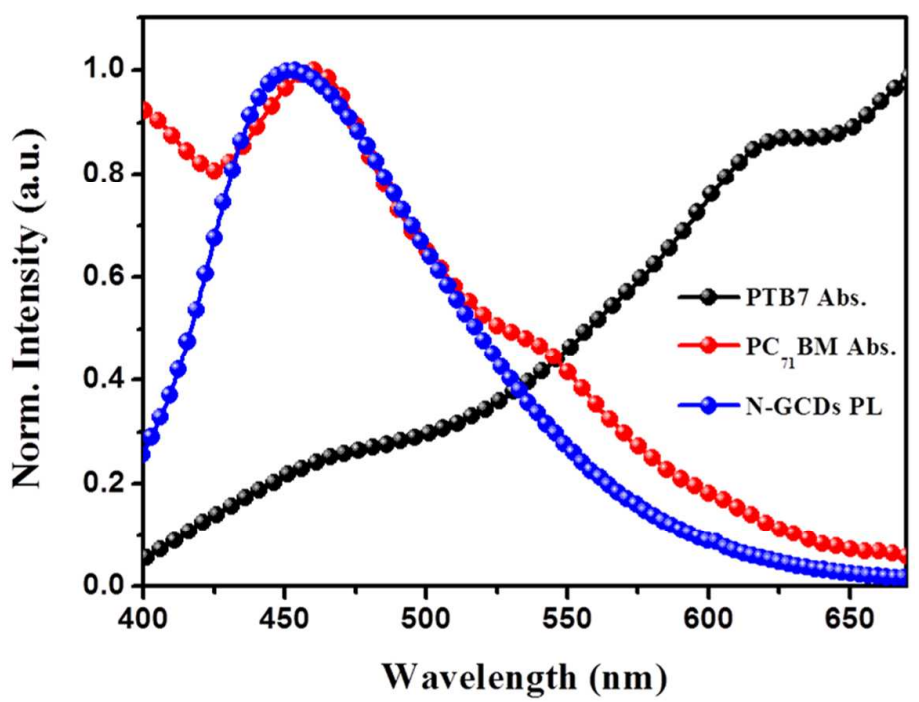

Fig. S11 Normalized absorption spectra of $\mathrm{PTB} 7$ and $\mathrm{PC}_{71} \mathrm{BM}$ samples and photoluminescence spectra of N-GCDs sample. 


\section{Time-resolved PL of N-GCDs.}

Table S1. PL lifetimes of the N-GCDs with $360 \mathrm{~nm}$ excitation wavelength.

\begin{tabular}{|c|c|c|c|c|c|c|c|c|}
\hline Sample $^{\mathrm{a})}$ & $\boldsymbol{A}_{\mathbf{1}}$ & $\tau_{\mathbf{1}}$ & $\boldsymbol{A}_{\mathbf{2}}$ & $\tau_{\mathbf{2}}$ & $\boldsymbol{A}_{\mathbf{3}}$ & $\tau_{\mathbf{3}}$ & $\tau_{\mathrm{av}}$ & $\chi^{2}$ \\
\hline N-GQDs & 0.104 & 1.789 & 0.085 & 5.244 & 0.026 & 14.107 & $7.86 \mathrm{~ns}$ & 1.012 \\
\hline
\end{tabular}

a) Monitored wavelength was $460 \mathrm{~nm}$. The PL decay curves were fitted by a triexponential function to calculate the lifetimes of the samples. The intensity weighted average exciton lifetime $\left(\tau_{\mathrm{avr}}\right)$ was $\left(\left(A_{1} \tau_{1}{ }^{2}+A_{2} \tau_{2}{ }^{2}+A_{3} \tau_{3}{ }^{2}\right) /\left(A_{1} \tau_{1}+A_{2} \tau_{2}+A_{3} \tau_{3}\right)\right) \cdot \chi^{2}$ is the reduced chi-squared value.

\section{Device performance of inverted polymer solar cells with/without N-GCDs.}

Table S2. Optimized photovoltaic response data for PTB7: $\mathrm{PC}_{71} \mathrm{BM}$ inverted solar cells with/without N-GCDs.

\begin{tabular}{ccccc}
\hline Sample & $\begin{array}{c}\boldsymbol{J}_{\text {SC }} \\
\left(\mathbf{m A} / \mathbf{c m}^{2}\right)\end{array}$ & $\begin{array}{c}\boldsymbol{V}_{\text {OC }} \\
(\mathbf{V})\end{array}$ & $\mathbf{F F}$ & $\begin{array}{c}\text { PCE (Avg.) } \\
(\%)\end{array}$ \\
\hline $\begin{array}{c}\text { Control (PTB7:PC } \\
\text { with 2 wt\% } \\
\text { N-GCDs }\end{array}$ & $15.3( \pm 0.11)$ & $0.73( \pm 0.002)$ & $0.65( \pm 0.02)$ & $7.3(7.1)$ \\
\hline
\end{tabular}


14. Stability tests of polymer solar cells with/without $\mathrm{N}-\mathrm{GCDs}$ in $\mathrm{PTB} 7: \mathrm{PC}_{71} \mathrm{BM}$ blend system under ambient conditions.

We examined the stability of the iPSCs with/without N-GCDs in PTB7:PC ${ }_{71} \mathrm{BM}$ blend system over 100 hours, which were not encapsulated and were stored in air under ambient conditions. As shown in Fig. S12, there is not much difference in air stability between control (without N-GCDs) and modified devices (with $2 \mathrm{wt} \% \mathrm{~N}$ GCDs).

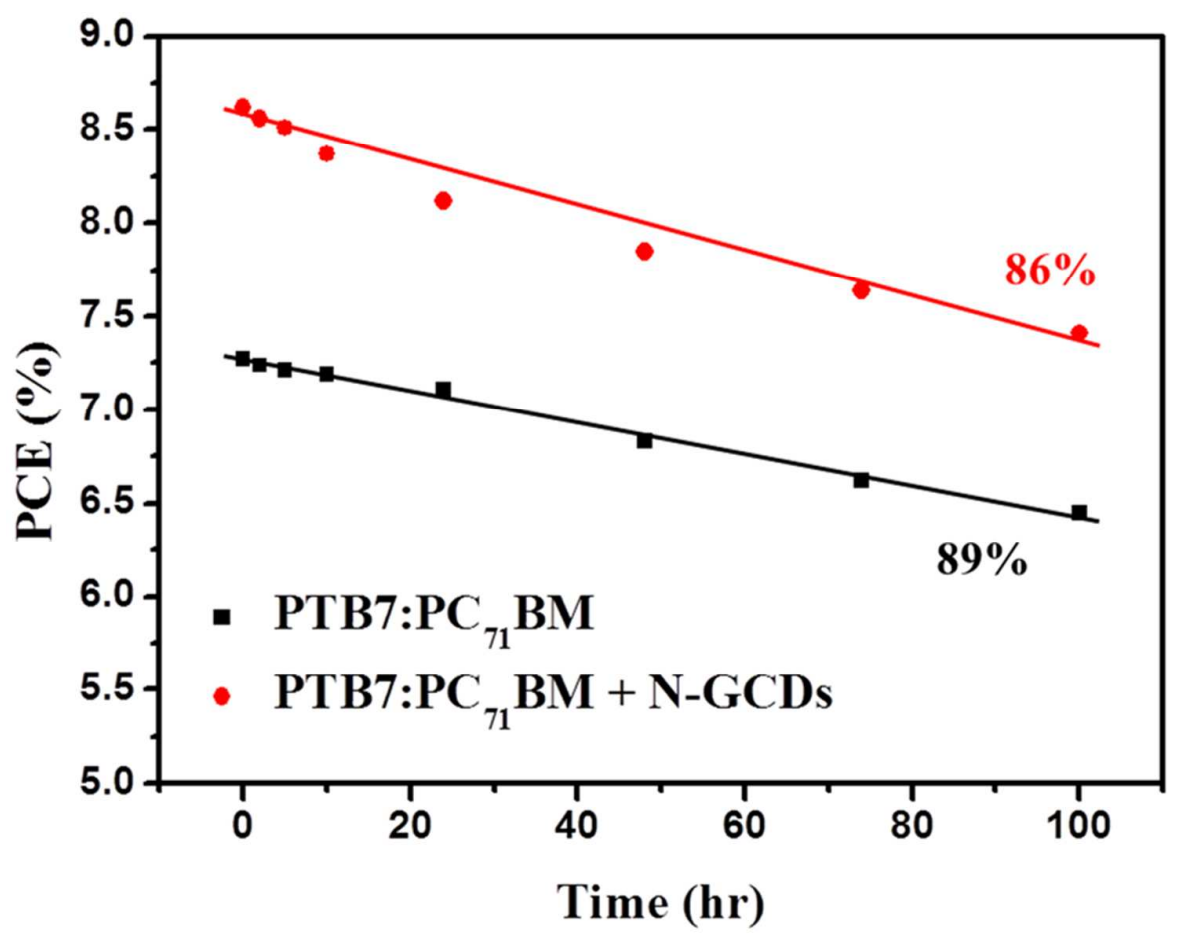

Fig. S12 PCEs as a function of storage time for unencapsulated PTB7:PC ${ }_{71} \mathrm{BM}$ inverted solar cells fabricated with/without N-GCDs in air under ambient conditions. 